\title{
DOSE-RESPONSE TO AEROSOLIZED KL 4 SURFACTANT IN THE SPONTANEOUSLY BREATHING CPAP-SUPPORTED PRETERM LAMB
}

\author{
M.R. Wolfson ${ }^{1,2}$, J. Wu ${ }^{3}$, T.L. Hubert ${ }^{3}$, J. Mazela ${ }^{4,5}$, T.J. Gregory ${ }^{5}$, R.G. Clayton ${ }^{5}$, T.H. Shaffer, ${ }^{6,7}$ \\ ${ }^{1}$ Physiology, Pediatrics and Medicine, ${ }^{2}$ Center for Inflammmation, Translational and Clinical Lung \\ Research, ${ }^{3}$ Physiology and Center for Inflammation, Translational, and Clinical Lung Research, Temple \\ University School of Medicine, Philadelphia, PA, USA, ${ }^{4}$ Poznan University, Poznan, Poland, ${ }^{5}$ Discovery \\ Laboratories, Inc., Warrington, ${ }^{6}$ Physiology \& Pediatrics, Temple University School of Medicine, \\ Philadelphiia, PA, ${ }^{7}$ Nemours Lung Research Ct, A.I. Dupont Hosp for Children, Wilmington, DE, USA
}

Background: We have demonstrated effectiveness of an aerosolized, peptide-containing, synthetic surfactant $\left(\mathrm{KL}_{4}\right.$ surfactant), in spontaneously breathing CPAP-supported preterm lambs. Dose-ranging studies are required to define the dose that produces optimal physiologic and biomarker responses.

Aims: To evaluate dose-responses to aerosolized $\mathrm{KL}_{4}$ surfactant using lung mechanics, histomorphology and lung inflammation biomarkers in spontaneously breathing, CPAP-supported preterm lambs.

Methods: Following Cesarean section, lambs ( $\mathrm{n}=20 ; 130-132$ days gestation) were anesthetized, instrumented, delivered, supported with $100 \%$ oxygen, CPAP, and caffeine then quasi-randomized to receive CPAP alone (controls) or CPAP plus aerosolized $\mathrm{KL}_{4}$ surfactant for up to 10, 20, 30 or 90 min. exposure at $1.2 \mathrm{~mL} / \mathrm{min}$. Cardiopulmonary parameters were monitored for $4 \mathrm{hrs}$. Lung IL-8 and histomorphometry were also measured.

Results: Compliance increased and lung IL-8 decreased in an aerosolized $\mathrm{KL}_{4}$ surfactant dose-dependent manner relative to CPAP treatment alone. Marked differences occurred with the 20 min dose vs. CPAP alone with little differences between the 20,30 and 90 min. doses. $\mathrm{PaO}_{2}$ was greater than in controls following the 10, 20 and $30 \mathrm{~min}$. doses and trended towards control values with the $90 \mathrm{~min}$. dose. Gross and histomorphometry lung assessment demonstrated greater and more homogenous expansion compared to controls, independent of treatment duration.

Conclusions: Aerosolized $\mathrm{KL}_{4}$ surfactant improved gas exchange, pulmonary mechanics, lung structure integrity, and reduced lung inflammation in a dose-dependent manner relative to treatment with CPAP alone. These observations provide preliminary guidance for titrating dosing strategies for optimal management of RDS.(Discovery Laboratories, Inc; DURIP-ONR; NIH P20 RR 020173). 